\title{
Consumo de Benzodiazepínicos relacionados a saúde mental nos últimos 10 anos: uma revisão integrativa
}

\author{
Mental health-related benzodiazepine use in the last 10 years: an integrative review \\ Uso de benzodiazepinas relacionadas con la salud mental en los últimos 10 años: una revisión \\ integradora
}

Recebido: 15/12/2021 | Revisado: 22/12/2021 | Aceito: 24/12/2021 | Publicado: 28/12/2021

\author{
Matheus Macedo Hernesto \\ ORCID: https://orcid.org/0000-0003-3527-2017 \\ Centro Universitário UNINOVAFAPI, Brasi \\ E-mail:hernestomatheus@ hotmail.com \\ Laura dos Santos Carneiro de Andrade \\ ORCID: https://orcid.org/0000-0002-2235-549X \\ Centro Universitário UNINOVAFAPI, Brasi \\ E-mail: laura.sca@hotmail.com \\ Maria José Santos Brandão Carvalho \\ ORCD: https://orcid.org/0000-0002-6191-3079 \\ Centro Universitário UNINOVAFAPI, Brasil \\ E-mail: mariabc2521@gmail.com \\ Isadora Machado Sanches \\ ORCID: https://orcid.org/0000-0001-5081-609X \\ Centro Universitário UNINOVAFAPI, Brasil \\ E-mail: sanches.isa6@ gmail.com \\ Lara Cavalcante Mourão \\ ORCD: https://orcid.org/ 0000-0003-4185-710X \\ Centro Universitário UNINOVAFAPI, Brasil \\ E-mail: lara.cavalcante.mourao@ gmail.com \\ Maria Carolina dos Santos da Trindade \\ ORCID: https://orcid.org/0000-0001-6619-3851 \\ Centro Universitário UNINOVAFAPI, Brasil \\ E-mail: carolinastrindade@hotmail.com \\ Lais Santos Dantas \\ ORCD: https://orcid.org/0000-0001-5592-4778 \\ Centro Universitário UNINOVAFAPI, Brasil \\ E-mail: lais_sdantas@hotmail.com \\ Karen Lima Barradas \\ ORCID: https://orcid.org/0000-0003-2489-0215 \\ Centro Universitário UNINOVAFAPI, Brasil \\ E-mail: k_barradas@yahoo.com
}

\begin{abstract}
Resumo
A saúde mental caracteriza-se como bem-estar cognitivo, comportamental e emocional. um estado de bem-estar em que um indivíduo uso de benzodiazepínicos (BDZs) representa uma questão crítica, pois um tratamento de longo prazo pode levar à dependência. Dessa forma a pesquisa teve por objetivo avaliar na literatura consumo de benzodiazepínicos relacionados a saúde mental nos anos de 2011 a 2021 . O presente estudo trata-se de uma pesquisa bibliográfica exploratória do tipo integrativa com natureza qualitativa. Os indivíduos com maior risco de abuso de BZD, particularmente são aqueles com histórico pessoal ou familiar de transtorno por uso de substâncias. O abuso de BZD pode ser dividido em dois padrões, incluindo abuso deliberado ou recreativo com a intenção de ficar chapado e abuso não intencional que começa como uso legítimo, mas depois evolui para uso impróprio. É fundamental verificar a saúde mental antes de prescrever, usar alternativas mais seguras e fazer intervenções apropriadas para combater o uso contínuo.
\end{abstract}

Palavras-chave: Benzodiazepínicos; Saúde mental; Abuso de medicamentos.

\footnotetext{
Abstract

Mental health is characterized as cognitive, behavioral and emotional well-being. a state of well-being in which an individual is using benzodiazepines (BDZs) represents a critical issue as long-term treatment can lead to dependence. Thus, the research aimed to evaluate in the literature consumption of benzodiazepines related to mental health in the years 2011 to 2021. The present study is an exploratory bibliographical research of the integrative type with a
} 
qualitative nature. Individuals most at risk for BZD abuse, particularly are those with a personal or family history of substance use disorder. BZD abuse can be divided into two patterns, including deliberate or recreational abuse with the intent to get high and unintentional abuse that starts out as legitimate use but then evolves into misuse. It is critical to check mental health before prescribing, use safer alternatives, and make appropriate interventions to combat continued use.

Keywords: Benzodiazepines; Mental health; Drug abuse.

\section{Resumen}

La salud mental se caracteriza por el bienestar cognitivo, conductual y emocional. un estado de bienestar en el que una persona está usando benzodiazepinas (BDZ) representa un problema crítico ya que el tratamiento a largo plazo puede conducir a la dependencia. Así, la investigación tuvo como objetivo evaluar en la literatura el consumo de benzodiacepinas relacionadas con la salud mental en los años 2011 a 2021 . El presente estudio es una investigación bibliográfica exploratoria de tipo integrativo con carácter cualitativo. Las personas con mayor riesgo de abuso de BZD, en particular son aquellas con antecedentes personales o familiares de trastorno por uso de sustancias. El abuso de BZD se puede dividir en dos patrones, incluido el abuso deliberado o recreativo con la intención de drogarse y el abuso no intencional que comienza como un uso legítimo pero luego evoluciona hacia un uso indebido. Es fundamental controlar la salud mental antes de recetar, utilizar alternativas más seguras y realizar las intervenciones adecuadas para combatir el uso continuo.

Palabras clave: Benzodiazepinas; Salud mental; Abuso de drogas.

\section{Introdução}

A saúde mental refere-se ao bem-estar cognitivo, comportamental e emocional. um estado de bem-estar em que um indivíduo percebe suas próprias habilidades, pode lidar com o estresse normal da vida, pode trabalhar de forma produtiva e é capaz de dar uma contribuição para sua comunidade (Murthy et al., 2017; Barry et al., 2019).

Dessa forma a Organização Mundial de Saúde (OMS) enfatiza que a saúde mental é "mais do que apenas a ausência de transtornos mentais ou deficiências". O auge da saúde mental não envolve apenas evitar condições ativas, mas também cuidar do bem-estar e felicidade contínuos. Eles também enfatizam que preservar e restaurar a saúde mental é crucial em uma base individual, bem como em diferentes comunidades e sociedades em todo o mundo (Tu et al., 2019).

Nesse contexto os benzodiazepínicos (BZD) constituem um grupo de psicotrópicos mais comumente utilizados na prática clínica devido às suas quatro ações principais: ansiolítico, hipnótico, anticonvulsivante e relaxante muscular. Geralmente, são indicados para transtornos de ansiedade, insônia e epilepsia. O uso de ansiolíticos e hipnóticos aumentou consideravelmente na última década (Dodds, 2017; Guina et al., 2018).

Em países desenvolvidos, como Austrália, França e Espanha, esses medicamentos são os mais prescritos e, entre eles, os benzodiazepínicos são os mais comuns e cerca de 20 milhões de prescrições são escritas todos os anos nos Estados Unidos e aproximadamente $10 \%$ da população relata ter usado benzodiazepínico como hipnótico (Balon et al., 2018).

No Brasil, pesquisa mostrou que os benzodiazepínicos foram a terceira substância mais consumida pelos 8.589 indivíduos entrevistados. Um estudo populacional com 1.606 participantes na cidade de Bambuí (Minas Gerais, Brasil) observou a frequência do uso desses medicamentos em aproximadamente $22 \%$ dos indivíduos com idade média de 69 anos, com prevalência no uso de benzodiazepínicos de longa ação e por mais de 12 meses (Noleto et al., 2016).

A eficácia desses medicamentos no tratamento de insônia e transtornos de saúde mental por um curto período de tempo é descrita na literatura. Porém, seu uso por longos períodos não é recomendado, principalmente, devido ao risco de desenvolver dependência e outros efeitos adversos (Bobes et al., 2012; Subramaniam et al., 2013).

O uso prolongado de benzodiazepínicos, mesmo em baixas dosagens, é fator de risco para o desenvolvimento de efeitos adversos que pode ser descrito como sonolência, vertigem, cansaço, confusão mental, dor de cabeça, ansiedade, letargia, ataxia, hipotensão postural, amnésia retrógrada, acidentes, tolerância, vício e aumento na frequência de quedas (Day \& Rosenthal., 2019). 
Apesar das evidências na literatura, os benzodiazepínicos são amplamente usados e comumente usados de forma inadequada. $\mathrm{O}$ abuso, insuficiência ou inadequação no uso desses medicamentos prejudica os usuários e contribui para o aumento dos gastos de recursos públicos, bem como para a irracionalidade no seu uso (Spagnolo et al, 2018; Quinn et al., 2019).

Segundo o uso de benzodiazepínicos pode exacerbar os transtornos mentais por uso de substâncias existentes ou se tornarem substâncias de abuso. Nesse sentido a presente pesquisa teve por objetivo avaliar na literatura consumo de benzodiazepínicos relacionados a saúde mental nos anos de 2011 a 2021.

\section{Metodologia}

O presente estudo trata-se de uma pesquisa bibliográfica exploratória do tipo integrativa com natureza qualitativa. Segundo Martins e Theóphilo (2016), tal pesquisa se torna necessária para o direcionamento de qualquer estudo científico, uma vez que busca descrever e discutir um assunto, tema ou problema mediante referências publicadas em livros, periódicos, revistas, enciclopédias, dicionários, jornais, sites, entre outras fontes.

A busca na literatura foi feita com base na consulta das bases de dados eletrônicas: Biblioteca Virtual em Saúde (BVS), Scientific Electronic Library Online (SciELO) Science Direct, Pubmed e Scopus, por meio dos seguintes Descritores em Ciências da Saúde (DeCS): Saúde Mental, Benzodiazepínicos, transtorno mental e medicamentos nos anos 2011 a 2021.

Com base na leitura dos artigos iniciais, foi feita uma seleção com base nos critérios inclusão: artigos originais, randomizados ou controlados, publicados no eixo temporal predefinido, nos idiomas inglês e português e que denotem a ingestão de benzodiazepínicos relacionados a transtornos de saúde mental.

As etapas de pesquisas tracejadas foram: estabelecimento dos fatores relevantes e dos objetivos da revisão bibliográfica; obtenção dos artigos (critérios de inclusão e exclusão); seleção e avaliação dos artigos.

Os dados foram apresentados em um quadro contendo as seguintes variáveis: Autor do artigo; ano de publicação; abordagem metodológica; periódico do artigo; objetivos do artigo e evidências do estudo. Após a coleta e leitura dos artigos, os mesmos serão organizados e separados em quadro, por meio de porcentagem simples e posteriormente, será feita a criação de duas categorias, realizando a discussão de acordo com os resultados dos artigos trabalhados.

\section{Resultados e Discussão}

Na busca realizadas nas quatro bases de dados: Biblioteca Virtual em Saúde (BVS), Scientific Electronic Library Online (SciELO) Science Direct, Pubmed e Scopus usando os descritores em conjunto foram encontrados um total de 639 artigos que após uma filtragem utilizando os critérios de inclusão e exclusão totalizaram em 12. O processo de filtragem se deu pelo fluxograma (Figura 1): 
Figura 1: Histograma da filtragem dos artigos.

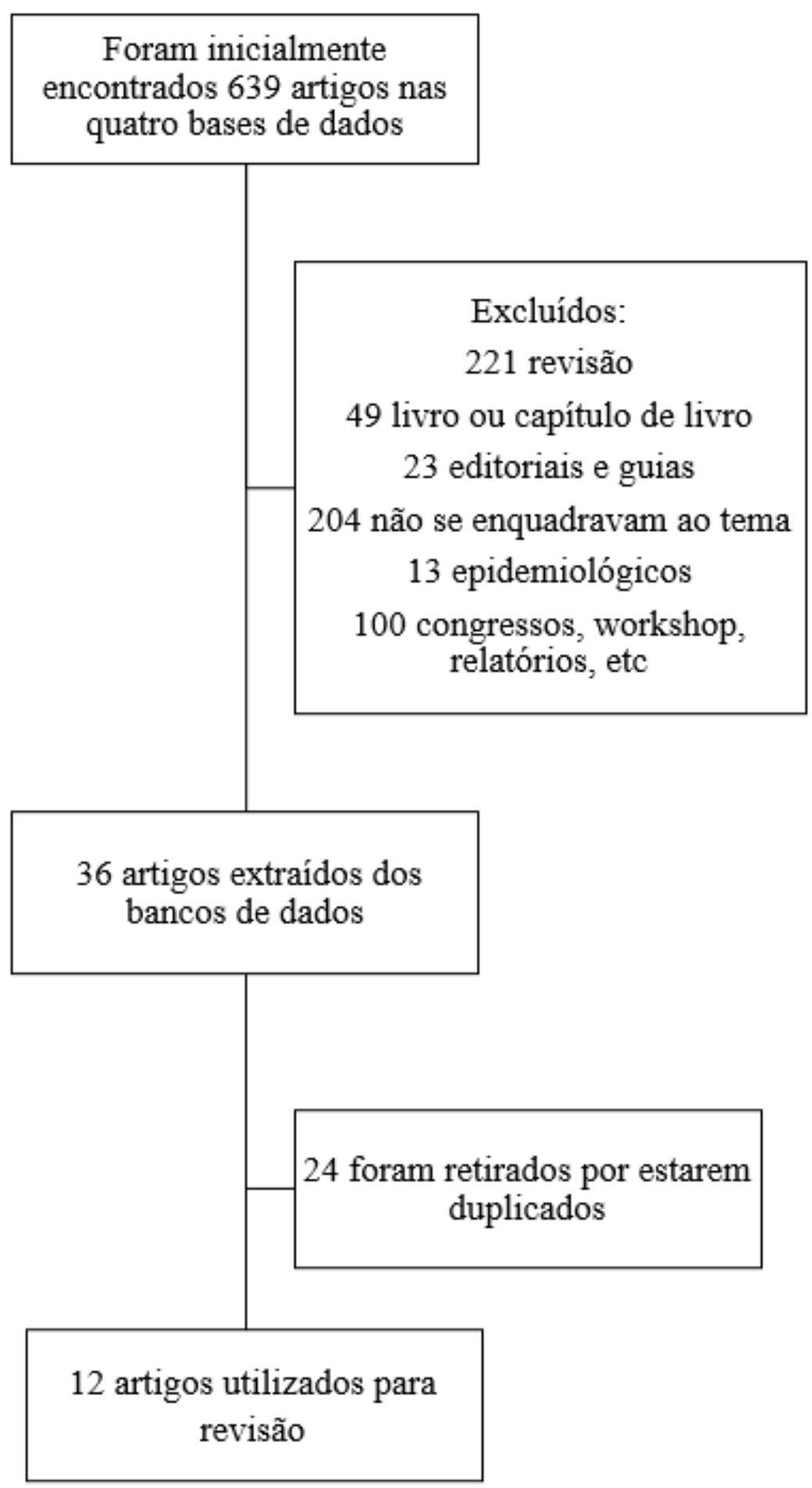

Fonte: Pesquisa dos autores.

A partir da coleta de dados no Quadro 1 estão distribuídos os resultados abordando consumo de benzodiazepínicos relacionados a saúde mental nos últimos 10 anos. 
Quadro 1: Estudos selecionados para a pesquisa.

\begin{tabular}{|c|c|c|c|c|}
\hline Autor/Ano & $\begin{array}{c}\text { Abordagem } \\
\text { metodológica }\end{array}$ & $\begin{array}{l}\text { Periódico } \\
\text { do artigo }\end{array}$ & Objetivos do artigo & Evidências do estudo \\
\hline $\begin{array}{c}\text { Bobes et al } \\
(2012)\end{array}$ & $\begin{array}{l}\text { Estudo prospectivo, não } \\
\text { controlado, não } \\
\text { intervencionista e } \\
\text { observacional de } 12 \\
\text { semanas em pacientes } \\
\text { com } 18 \text { anos ou mais }\end{array}$ & $\begin{array}{l}\text { European } \\
\text { psychiatry }\end{array}$ & $\begin{array}{l}\text { Avaliar a eficácia e } \\
\text { pregabalina no torabilidade da } \\
\text { descontinuação dos benzodiazepínicos } \\
\text { em usuários de longo prazo. }\end{array}$ & $\begin{array}{l}\text { Resultados sugerem que a pregabalina é um } \\
\text { tratamento adjuvante eficaz e bem tolerado } \\
\text { para a suspensão de benzodiazepínicos em } \\
\text { pacientes com transtornos de saúde mental. }\end{array}$ \\
\hline $\begin{array}{l}\text { Subramaniam } \\
\text { et al (2013) }\end{array}$ & $\begin{array}{l}\text { Estudo transversal com } \\
\text { entrevistas presenciais }\end{array}$ & $\begin{array}{l}\text { BMC } \\
\text { psychiatry }\end{array}$ & $\begin{array}{l}\text { Estabelecer a prevalência e os fatores } \\
\text { associados ao uso de antidepressivos e } \\
\text { benzodiazepínicos. }\end{array}$ & $\begin{array}{l}\text { Descobriu-se que o uso de antidepressivos e } \\
\text { benzodiazepínicos na população era } \\
\text { relativamente baixo, e 'busca de ajuda' foi o } \\
\text { preditor mais importante do uso. }\end{array}$ \\
\hline $\begin{array}{l}\text { Naloto et al } \\
\text { (2016) }\end{array}$ & $\begin{array}{c}\text { Estudo transversal com } \\
\text { coleta de dados de } \\
\text { pacientes atendidos no } \\
\text { Ambulatório Municipal de } \\
\text { Saúde Mental }\end{array}$ & $\begin{array}{l}\text { Ciencia \& } \\
\text { saude } \\
\text { coletiva }\end{array}$ & $\begin{array}{l}\text { comparar as prescrições de } \\
\text { benzodiazepínicos para adultos e idosos } \\
\text { quanto ao indicador de uso adequado. }\end{array}$ & $\begin{array}{l}\text { O uso crônico foi observado em todos os } \\
\text { adultos e idosos com transtornos } \\
\text { depressivos e de ansiedade e uma minoria } \\
\text { de prescrições para adultos e idosos era } \\
\text { apropriada. }\end{array}$ \\
\hline $\begin{array}{l}\text { Spagnolo et al } \\
\text { (2018) }\end{array}$ & $\begin{array}{l}\text { Estudo de caso com uma } \\
\text { amostra intencional de } 18 \\
\text { pacientes }\end{array}$ & & $\begin{array}{l}\text { Verificar o treinamento com base } \\
\text { no Guia de Intervenção do Programa de } \\
\text { Ação de Saúde Mental }\end{array}$ & $\begin{array}{l}\text { Os resultados podem ser usados para } \\
\text { adaptar fatores estruturais, organizacionais, } \\
\text { do provedor, do paciente e de inovação } \\
\text { antes de futuras implementações do } \\
\text { treinamento baseado em Programa de Ação } \\
\text { de Saúde Mental na Tunísia }\end{array}$ \\
\hline $\begin{array}{l}\text { Bushnell, } \\
\text { Crystal \& } \\
\text { Olfson (2019) }\end{array}$ & $\begin{array}{l}\text { Estudo de coorte } \\
\text { retrospectivo }\end{array}$ & $\begin{array}{l}\text { American } \\
\text { journal of } \\
\text { preventive } \\
\text { medicine }\end{array}$ & $\begin{array}{l}\text { Descrever os jovens que iniciaram o } \\
\text { tratamento com prescrição de } \\
\text { benzodiazepínicos, identificar possíveis } \\
\text { indicações e preocupações com a } \\
\text { prescrição, estimar a duração do } \\
\text { tratamento por indicação e saúde mental. }\end{array}$ & $\begin{array}{l}\text { Crianças e adolescentes recebem prescrição } \\
\text { de benzodiazepínicos para vários problemas } \\
\text { de saúde mental e outras condições médicas, } \\
\text { muitos deles sem evidências de eficácia } \\
\text { pediátrica. O }\end{array}$ \\
\hline $\begin{array}{c}\text { Day \& } \\
\text { Rosenthal } \\
(2019)\end{array}$ & Estudo transversal & $\begin{array}{l}\text { Drug and } \\
\text { alcohol } \\
\text { dependenc } \\
\text { e }\end{array}$ & $\begin{array}{l}\text { Examinar a associação entre variáveis de } \\
\text { isolamento social (morar sozinho, ser } \\
\text { solteiro e não frequentar serviços } \\
\text { religiosos) e o uso indevido de opióides / } \\
\text { benzodiazepínicos prescritos em idosos. }\end{array}$ & $\begin{array}{l}\text { Aumento da probabilidade de uso indevido } \\
\text { de medicamentos prescritos de opióides e } \\
\text { benzodiazepínicos combinados foi } \\
\text { observada entre adultos mais velhos } \\
\text { solteiros }\end{array}$ \\
\hline $\begin{array}{l}\text { Quinn et al } \\
\text { (2019) }\end{array}$ & Estudo transversal & $\begin{array}{l}\text { BMC } \\
\text { public } \\
\text { health }\end{array}$ & $\begin{array}{l}\text { Estimou associações de diversos } \\
\text { diagnósticos pré-existentes de saúde } \\
\text { mental, histórico de saúde mental dos } \\
\text { pais e socioeconômico na infância com } \\
\text { padrões de prescrição de medicamnetos. }\end{array}$ & $\begin{array}{l}\text { Entre } 1.482 .462 \text { adolescentes e adultos } \\
\text { jovens, as taxas de iniciação e } \\
\text { medicamentos foram maiores para aqueles } \\
\text { com histórico de saúde mental dos pais. }\end{array}$ \\
\hline $\begin{array}{l}\text { Campanha et } \\
\text { al (2020) }\end{array}$ & Estudo transversal & Clinics & $\begin{array}{l}\text { Relatar a prevalência e os fatores } \\
\text { associados ao uso de benzodiazepínicos } \\
\text { na população em geral e em portadores } \\
\text { de transtorno mental }\end{array}$ & $\begin{array}{l}\text { Os achados fornecem uma visão geral do } \\
\text { uso de benzodiazepínicos na população em } \\
\text { geral, que serão úteis no domínio da saúde } \\
\text { pública. O uso de benzodiazepínicos foi } \\
\text { maior em pessoas com transtorno mental, } \\
\text { sendo as pessoas com transtorno do humor } \\
\text { as mais vulneráveis }\end{array}$ \\
\hline $\begin{array}{l}\text { Mayberry, } \\
\text { Nechuta \& } \\
\text { Krishnaswami } \\
(2021) \\
\end{array}$ & $\begin{array}{l}\text { Estudo transversal com } \\
\text { coleta de dados de } \\
\text { pacientes }\end{array}$ & $\begin{array}{l}\text { Journal of } \\
\text { Substance } \\
\text { Abuse } \\
\text { Treatment } \\
\end{array}$ & $\begin{array}{l}\text { Avaliou cada paciente um ano após a } \\
\text { overdose inicial para determinar a } \\
\text { probabilidade de overdose de repetição. }\end{array}$ & $\begin{array}{l}\text { A pesquisa mostrou que os } \\
\text { benzodiazepínicos e os transtornos mentais } \\
\text { podem aumentar a probabilidade de } \\
\text { overdose de repetição }\end{array}$ \\
\hline $\begin{array}{l}\text { Hawkins et al } \\
\quad(2021)\end{array}$ & $\begin{array}{l}\text { Estudo qualitativo por } \\
\text { meio de entrevistas } \\
\text { semiestruturadas }\end{array}$ & $\begin{array}{l}\text { Pain } \\
\text { Medicine }\end{array}$ & $\begin{array}{l}\text { Examinar as perspectivas dos provedores } \\
\text { sobre opióides e benzodiazepínicos } \\
\text { coprescritos e identificamos as barreiras } \\
\text { e facilitadores para a desprescrição. }\end{array}$ & $\begin{array}{l}\text { Desafios de coordenação entre os } \\
\text { prescritores, as preocupações com o tempo } \\
\text { insuficiente e a resistência dos pacientes em } \\
\text { descontinuar esses medicamentos precisam } \\
\text { ser resolvidos para que os esforços sejam } \\
\text { bem-sucedidos. }\end{array}$ \\
\hline $\begin{array}{l}\text { Olopoenia et } \\
\text { al (2021) }\end{array}$ & $\begin{array}{l}\text { Estudo de coorte } \\
\text { retrospectivo }\end{array}$ & $\begin{array}{l}\text { Drug and } \\
\text { alcohol } \\
\text { dependence }\end{array}$ & \begin{tabular}{lrrr} 
Descrever & \multicolumn{2}{c}{ padrões } & \multicolumn{2}{c}{ específicos } \\
associados a & co-prescrições & de \\
gabapentina, & \multicolumn{2}{c}{ opióides } & e \\
benzodiazepínicos & entre & beneficiários \\
deficientes do Medicare. & &
\end{tabular} & $\begin{array}{l}\text { Dadas as preocupações de segurança } \\
\text { associadas à gabapentina, opioides e } \\
\text { benzodiazepínicos, é imperativo que os } \\
\text { benefícios e riscos da co-prescrição desses } \\
\text { medicamentos sejam examinados de forma } \\
\text { abrangente, especialmente para aqueles em } \\
\text { subgrupos vulneráveis. }\end{array}$ \\
\hline $\begin{array}{l}\text { Lee et al } \\
(2021)\end{array}$ & Estudo transversal & $\begin{array}{l}\text { Addictive } \\
\text { Behaviors }\end{array}$ & $\begin{array}{l}\text { Verificar o uso de nicotina (tabagismo e } \\
\text { outros usos do tabaco) estaria } \\
\text { positivamente associado ao uso indevido } \\
\text { de benzodiazepínicos entre adultos, } \\
\text { mesmo após o controle de fatores de } \\
\text { confusão demográficos e clínicos. }\end{array}$ & $\begin{array}{l}\text { A nicotina está independentemente } \\
\text { associada ao uso indevido de } \\
\text { benzodiazepínicos, mesmo após o controle } \\
\text { do uso de outras drogas e variáveis } \\
\text { psiquiátricas. Estudos futuros examinando } \\
\text { os mecanismos potenciais que ligam o uso } \\
\text { de nicotina e benzodiazepínicos são } \\
\text { necessários. }\end{array}$ \\
\hline
\end{tabular}

Fonte: Pesquisa dos autores. 


\subsection{Abuso de benzodiazepínicos e saúde mental}

O uso indevido e o abuso de benzodiazepínicos são um problema crescente, onde segundo Naloto et al (2016) aproximadamente $2,3 \%$ a $18 \%$ das pessoas usaram indevidamente sedativos ou tranquilizantes para uso não médico durante a vida e quase $10 \%$ desses indivíduos preencheram os critérios para abuso ou dependência.

Em 2010, havia cerca de 186.000 novos abusadores de BZD em que departamentos de emergência viram um aumento acentuado de 139\% nas visitas relacionadas com BZD (Bobes et al., 2012). A idade avançada e a presença de outros medicamentos foram associadas a desfechos mais graves, incluindo óbito. O número de admissões em programas de tratamento para abuso de BZD quase triplicou de 1998 a 2008. Durante esse mesmo período, o número de todas as admissões em programas de tratamento de abuso de substâncias aumentou apenas 11\% (Subramaniam et al., 2013; Naloto et al., 2016)

Mayberry et al. (2021) enfatizaram que existe um subconjunto de indivíduos com maior risco de abuso de BZD, particularmente aqueles com histórico pessoal ou familiar de transtorno por uso de substâncias. O abuso de BZD pode ser dividido em dois padrões, incluindo abuso deliberado ou recreativo com a intenção de ficar chapado e abuso não intencional que começa como uso legítimo, mas depois evolui para uso impróprio (Campanha et al., 2020).

Os benzodiazepínicos são comumente prescritos para pacientes com doenças mentais graves, como esquizofrenia e transtorno bipolar, mas seu uso no contexto de transtornos por uso de substâncias concomitantes é altamente controverso, tais medicamentos também são amplamente prescritos porque são eficazes no controle dos sintomas psiquiátricos e dos efeitos colaterais dos medicamentos (Hawkins et al., 2021).

Por outro lado, como os benzodiazepínicos podem exacerbar transtornos por uso de substâncias existentes ou se tornarem substâncias de abuso, muitos especialistas recomendam evitar a prescrição de benzodiazepínicos para pacientes com transtornos concomitantes (Day \& Rosenthal, 2019).

No estudo realizado por Bushnell et al. (2019) foi visualizado uma alta taxa de uso de benzodiazepínicos prescritos (43 por cento) que sugere que muitos médicos estão dispostos a prescrever benzodiazepínicos para pacientes com doenças concomitantes. Benzodiazepínicos foram prescritos ao longo do estudo, principalmente para pacientes que apresentavam altos níveis de sintomas em geral e sintomas afetivos (ansiedade e depressão) em particular, bem como baixa qualidade de vida.

Esta alta taxa de prescrição de benzodiazepínicos para pessoas com doença mental grave e transtorno por uso de substâncias foi recentemente replicada em um estudo muito maior, que mostrou que 62,5\% receberam prescrições de benzodiazepínicos (Hawkins et al., 2021).

A evidência de pesquisa a respeito do abuso de benzodiazepínicos entre pessoas com transtornos por uso de substâncias na população em geral é limitada, e alguns especialistas argumentam que esses medicamentos podem ser usados com segurança com supervisão (Campanha et al., 2020).

$\mathrm{Na}$ pesquisa proposta por Mayberry et al. (2021) o uso de benzodiazepínicos prescritos não estava relacionado ao abuso de substâncias e aos desfechos de hospitalização. Os pacientes que tomaram benzodiazepínicos prescritos geralmente melhoraram e tiveram resultados semelhantes aos dos pacientes para os quais os benzodiazepínicos não foram prescritos, embora nenhum grupo tenha melhorado em termos de sintomas afetivos e o grupo dos benzodiazepínicos experimentou sintomas afetivos e gerais consistentemente piores.

Embora estudos controlados sejam necessários para resolver definitivamente a questão dos efeitos dos benzodiazepínicos nos resultados para pessoas com distúrbios concomitantes, os resultados relatados por Mayberry et al. (2021) contrastam a clozapina um BZD estava associada a reduções dramáticas no abuso de substâncias - uma descoberta que foi replicada em outros estudos naturalísticos e que levou a testes controlados. 
No estudo de Lee et al (2021) pacientes com diagnóstico duplo para os quais foram prescritos benzodiazepínicos tinham mais de duas vezes mais chances de desenvolver abuso de benzodiazepínicos do que aqueles que não usaram benzodiazepínicos prescritos (15\% em comparação com 6\%).

Assim, a exposição aos benzodiazepínicos prescritos pode aumentar o risco de abuso de benzodiazepínicos ou, dito de outra forma, as prescrições médicas podem criar outro problema de abuso para este grupo de indivíduos já altamente vulneráveis. Entre aqueles para os quais foram prescritos benzodiazepínicos, níveis elevados de sintomas afetivos foram o único preditor do desenvolvimento de abuso de benzodiazepínicos (Olopoenia et al., 2021).

\subsection{Características clinicas e Benzodiazepínicos}

O uso de benzodiazepínicos também variou com as características clínicas dos pacientes. Conforme relatado nos estudos, os psiquiatras eram mais propensos a prescrever benzodiazepínicos para pacientes com níveis mais altos de comorbidade médica, talvez porque esses pacientes são vistos com mais frequência ou têm níveis mais elevados de angústia.

Os médicos também eram mais propensos a prescrever benzodiazepínicos para pacientes com transtornos de ansiedade concomitantes, que poderiam sentir mais alívio dos sintomas com esses medicamentos, e menos propensos a prescrever esses medicamentos para pacientes com transtornos por uso simultâneo de substâncias, que poderiam experimentar um uso mais problemático. No entanto, nesta amostra, uma porcentagem substancial (30\%) de pacientes deprimidos com transtornos por uso de substâncias comórbidos receberam benzodiazepínicos em regime ambulatorial, um achado que suscita preocupação.

Embora no estudo de Noleto et al (2016) os autores tenham encontrado uma associação modesta entre o uso de benzodiazepínicos e o aumento das taxas de hospitalização ajustadas para a idade, é surpreendente que também tenhamos verificado que o uso de benzodiazepínicos foi associado a um maior aumento de hospitalização entre os usuários mais jovens, em comparação com os usuários mais velhos.

No entanto, com um desenho de estudo transversal, não é possível desagregar completamente as potenciais interrelações entre o uso de benzodiazepínicos, gravidade da doença e hospitalização. Portanto, essas associações devem ser interpretadas com cautela. Estudos longitudinais e de caso-controle que examinam a relação entre os benzodiazepínicos e os resultados de saúde, como comprometimento cognitivo, quedas e fraturas de quadril, geralmente, mas nem sempre, relatam resultados piores entre usuários de benzodiazepínicos de longo prazo (Spagnolo et al., 2018; Olopoenia et al., 2021).

Tanto os BZDs ansiolíticos quanto os hipnóticos pareciam ser usados principalmente por pacientes com escolaridade intermediária. Esse achado pode ser explicado pelo fato de a amostra em estudo incluir, principalmente, sujeitos com nível de escolaridade médio-alto (Subramaniam et al., 2013).

Além disso, são necessários estudos que avaliem BZDs hipnóticos e ansiolíticos separadamente e, quando possível, cada BZD per se. Do ponto de vista clínico, BZD devem ser prescritos com base em seus perfis farmacocinéticos e farmacodinâmicos em que um tratamento medicamentoso pode modificar o curso, as características e a capacidade de resposta da doença para a qual foi administrado (isto é, comorbidade iatrogênica) (Day; Rosenthal., 2019).

\section{Conclusão}

Observando os achados da literatura, é possível verificar que parte dos resultados encontrados se assemelham a outras cidades brasileiras e até mesmo países, demonstrando a necessidade de iniciativas de planejamento de intervenções que visem o uso adequado desses medicamentos e que, consequentemente, beneficiem os pacientes. 
O incentivo ao aprimoramento dos profissionais envolvidos no cuidado a esses pacientes e à acurácia na prescrição dos benzodiazepínicos podem ser algumas medidas adotadas com o objetivo de proporcionar o uso adequado desses psicotrópicos neste serviço de saúde.

$\mathrm{O}$ abuso de medicamentos prescritos atingiu níveis epidêmicos. Os esforços atuais para reduzir a morbidade e mortalidade associadas não tiveram sucesso. As taxas continuam subindo. Pesquisas futuras devem ser conduzidas para entender melhor os fatores de risco para o abuso de BZD.

É fundamental verificar a saúde mental antes de prescrever, usar alternativas mais seguras e fazer intervenções apropriadas para combater o uso contínuo. O aumento dos programas de tratamento do abuso de substâncias, bem como o financiamento para esses programas no futuro, será um componente essencial na luta contra o problema crescente.

Pesquisas empíricas futuras são necessárias para avaliar os efeitos dos BZDs, com ênfase em transtornos de saúde mental. De modo geral, quando usados conforme orientação de um médico, os benzodiazepínicos são relativamente seguros por curtos períodos de tempo.

\section{Referências}

Balon, R., Chouinard, G., Cosci, F., Dubovsky, S. L., Fava, G. A., Freire, R. C., \& Weintraub, S. J. (2018). International task force on benzodiazepines. Psychotherapy and psychosomatics, 87(4), 193-195.

Barry, M. M., Clarke, A. M., Petersen, I., \& Jenkins, R. (2019). Implementing mental health promotion. Springer Nature.

Bobes, J., Rubio, G., Teran, A., Cervera, G., López-Gómez, V., Vilardaga, I., \& Pérez, M. (2012). Pregabalin for the discontinuation of long-term benzodiazepines use: an assessment of its effectiveness in daily clinical practice. European psychiatry, 27(4), 301-307.

Bushnell, G. A., Crystal, S., \& Olfson, M. (2019). Prescription benzodiazepine use in privately insured US children and adolescents. American journal of preventive medicine, 57(6), 775-785.

Campanha, A. M., Ravagnani, B., Milhorança, I. A., Bernik, M. A., Viana, M. C., Wang, Y. P., \& Andrade, L. H. (2020). Benzodiazepine use in Sao Paulo, Brazil. Clinics, 75 .

Day, B. F., \& Rosenthal, G. L. (2019). Social isolation proxy variables and prescription opioid and benzodiazepine misuse among older adults in the US: A cross-sectional analysis of data from the National Survey on Drug Use and Health, 2015-2017. Drug and alcohol dependence, $204,107518$.

Dodds, T. J. (2017). Prescribed benzodiazepines and suicide risk: a review of the literature. The primary care companion for CNS disorders, 19(2), 0-0.

Guina, J., \& Merrill, B. (2018). Benzodiazepines I: upping the care on downers: the evidence of risks, benefits and alternatives. Journal of clinical medicine, 7(2), 17.

Hawkins, E. J., Lott, A. M., Danner, A. N., Malte, C. A., Hagedorn, H. J., Berger, D., \& Saxon, A. J. (2021). Primary Care and Mental Health Prescribers, Key Clinical Leaders, and Clinical Pharmacist Specialists' Perspectives on Opioids and Benzodiazepines. Pain Medicine.

Lee, J., Salloum, R. G., Lindstrom, K., \& McHugh, R. K. (2021). Benzodiazepine misuse and cigarette smoking status in US adults: Results from the National Survey on Drug Use and Health, 2015-2018. Addictive Behaviors, 123, 107058.

Martins, G. D. A., \& Theóphilo, C. R. (2016). Metodologia da investigação científica para as ciências sociais. 3. São Paulo: Atlas.

Mayberry, S., Nechuta, S., \& Krishnaswami, S. (2021). Impact of benzodiazepines and polysubstance status on repeat non-fatal drug overdoses. Journal of Substance Abuse Treatment, 123, 108285.

Murthy, R. S. (2017). National mental health survey of India 2015-2016. Indian journal of psychiatry, 59(1), 21.

Naloto, D. C. C., Lopes, F. C., Barberato Filho, S., Lopes, L. C., Del Fiol, F. D. S., \& Bergamaschi, C. D. C. (2016). Prescription of benzodiazepines for adults and older adults from a mental health clinic. Ciencia \& saude coletiva, 21, 1267-1276.

Olopoenia, A., Camelo-Castillo, W., Qato, D. M., Adekoya, A., Palumbo, F., Sera, L., \& Simoni-Wastila, L. (2021). Patterns of opioid and benzodiazepine use with gabapentin among disabled Medicare beneficiaries-A retrospective cohort study. Drug and alcohol dependence, 109180.

Quinn, P. D., Rickert, M. E., Franck, J., Sariaslan, A., Boersma, K., Lichtenstein, P., \& D’Onofrio, B. M. (2019). Associations of mental health and family background with opioid analgesic therapy: a nationwide Swedish register-based study. Pain, 160(11), 2464.

Spagnolo, J., Champagne, F., Leduc, N., Melki, W., Piat, M., Laporta, M., \& Charfi, F. (2018). "We find what we look for, and we look for what we know": factors interacting with a mental health training program to influence its expected outcomes in Tunisia. BMC public health, 18(1), 1-19.

Subramaniam, M., He, V. Y., Vaingankar, J. A., Abdin, E., \& Chong, S. A. (2013). Prevalence of and factors related to the use of antidepressants and benzodiazepines: results from the Singapore Mental Health Study. BMC psychiatry, 13(1), 1-10.

Tu, T. T. H., Abiko, Y., \& Toyofuku, A. (2019). Mental health disorders. British Dental Journal, 227(12), $1010-1010$. 